\title{
PSEUDO POWER SIGNATURES FOR AIRCRAFT FAULT DETECTION AND IDENTIFICATION
}

\author{
Min Luo, Louisiana State University, Baton Rouge, $L A^{1}$ \\ Jorge L. Aravena, Louisiana State University, Baton Rouge, LA ${ }^{1}$ \\ Fahmida N. Chowdhury, University of Louisiana, Lafayette, LA ${ }^{1}$
}

\begin{abstract}
:
In earlier work, we presented proof of concept for the use of signal processing tools for fast fault detection in a model free framework. In this paper, we elaborate on the concept by applying pseudo power signatures to detect faults. We introduce a Singular Value Decomposition (SVD) approach for the computation of pseudo power signatures and discuss some of the advantages of the approach. In particular, we expand the concept of pseudo power signatures to that of signature subspaces. New experimental data from a reliable B747 aircraft model are used to test the proposed schemes.
\end{abstract}

\section{Introduction}

The problem of fault detection and identification has been extensively studied for many years. From a purely statistical point of view, Wald's Sequential Probability Test Ratio [1] can be considered a significant contribution to the development of on-line techniques. One should also mention the work of Basseville and Nikiforov [2] and the excellent survey of detection of change algorithms. Most of the fault detection methods available in the literature $[3,4,5]$ are residual-based where one has a model for the system and can compare the actual output with those produced by the model. Also their input is noise free. If a system model is not available, many researchers advocate building a model using measured input-output data. In contrast, our current research is focused on those practical situations where (1) reliable models are not available (2) the inputs are not measurable (3) the inputs are not noise free.
In such situations, one must use only the output data supplied by sensors. Our contention is that one can use signal-processing techniques on sensor data and enhance the effect of the fault, i.e., make it more apparent to the operator. In [6] we considered the special case of power transmission systems and proposed a model free approach based on filter bank processing and the definition of "instantaneous information vectors" for fault detection and identification. In [7] we showed that a filter bank can separate a sensor signal into several orthogonal components and permits the definition of narrow frequency bands where the effect of a fault is easily seen. But the decomposition does not lend itself easily to the implementation of an automatic detection of change algorithm.

In this paper we propose to use the pseudo power signature and some of its extensions to develop detectors of change. Our application of interest is aircraft safety and our test bed is NASA's model for a B747 aircraft implemented in the DASMAT environment by Gary Balas of the University of Minnesota [8].

\subsection{Mathematical Preliminaries}

Over the past years, extensive research has been done to the subject of time-frequency distributions for analysis of non-stationary signals. In most cases, they describe the energy distribution of the signal in a particular time-frequency region.

Continuous Wavelet Transform (CWT) was designed to provide variable resolution over the time-frequency plane. The shifted and dilated wavelets $\varphi_{a, b}$ provide a natural multiresolution of a

\footnotetext{
${ }^{1}$ Supported in part under grants NASA NCC5-573, contract NASA/LEQSF(2001-04)-01
} 
given function $x(t) \in L^{2}(R)$ in time and frequency through the use of the time index parameter $b$ and the scale parameter $a$. In general, the scale $a$ is inversely proportional to frequency, which implies that large scales correspond to low frequencies in the signal, and vice versa.

The scalogram of a function $x(t)$ with CWT $c_{\varphi}^{x}(a, b)$ is defined as

$S C_{\varphi}^{x}(a, b)=\left|c_{\varphi}^{x}(a, b)\right|^{2}$

The signal energy over a given support, $R$, is defined as

$E_{\varphi}^{x(R)}=C_{\varphi}^{-1} \iint_{(a, b) \in R} S C_{\varphi}^{x}(a, b) \frac{d b d a}{a^{2}}$

Hence, the scalogram can be used as a timefrequency energy density function.

\subsection{Pseudo Power Signature}

The basic idea is to develop a signature that characterizes the energy distribution of a signal in a manner that is essentially independent of the duration of the signal. We create this signature by defining an "instantaneous energy distribution" which is called the pseudo power signature.

The ideal situation would arise if one could define a wavelet such that for a given class of signals the corresponding wavelet transforms are separable, i.e.

$c_{\varphi}^{x}(a, b)=s_{\varphi}^{x}(a) r_{\varphi}^{x}(b)$

For any time interval $t_{1}<b<t_{2}$, the energy of the signal would be given by

$E\left(t_{1}, t_{2}\right)=\int_{a} \frac{\left|s_{\varphi}^{x}(a)\right|^{2}}{a^{2}} d a \int_{t_{1}}^{t_{2}}\left|r_{\varphi}^{x}(b)\right|^{2} d b$

Hence, the signal $\left|s_{\varphi}^{z}(a)\right|^{2}$, suitably normalized, becomes a scale power density function that is independent of time - a power signature. Moreover, for wavelets with compact support, the evaluation of the CWT depends only the values of the signal in a neighborhood of the time parameter (especially for small scales). Hence the signature can become essentially independent of the duration of the signal or the segment available.

Since it has been shown that no admissible wavelet will lead to a separable transform for an energy signal, one needs to resort to decompositions that satisfy Eq. (3) in an approximate way.

We note for future reference that a direct generalization of pseudo power signatures arises when one requires separability only for a set of scales. In this case, Eq. (4) will hold only for that range of scales and one can define a restricted scale power signature.

\section{The Matrix SVD Approach}

This section will present an approach to generate power signature for different signal classes using Singular Value Decomposition (SVD) of finite dimensional matrices. The methodology is based on a principal component analysis technique, and is derived from the decomposition of the CWT of a signal as a sum of separable terms. This analysis is based on the following results [9].

Proposition 2.1 The CWT can always be expressed as

$c_{\varphi}^{x}(a, b)=\sum_{i} \sigma_{i} s_{i}(a) r_{i}(b)$

$s_{i}(a) \in S=L^{2}\left(R, C_{\varphi}^{-1} \frac{d a}{a^{2}}\right), r_{i}(b) \in L^{2}(R, d b)$ for

each $i$. The function sets $\left\{s_{i}\right\}_{i}$ and $\left\{r_{i}\right\}_{i}$ are complete in $S$ and $L^{2}$ respectively.

The principal component of $c_{\varphi}^{x}$, denoted by $P C\left[c_{\varphi}^{x}\right]$, is given by $\sigma_{1} s_{1}(a) r_{1}(b)$. The function $s_{1}$ can then be used to define the pseudo power signature for the associated signal $x$.

In essence, Proposition 2.1 provides a decomposition of the CWT function $c_{\varphi}^{x}(a, b)$ as a sum of separable terms. This decomposition is similar to the more commonly known SVD applied to finite dimensional matrices.

Significant computational simplification is achieved if the determination of pseudo power 
signatures is carried out for finite discrete signal sets and can be reduced to a standard matrix SVD problem. In [9], the author showed that under some reasonable smoothness conditions, a discretization of the CWT in both time and scale yields a suitable matrix that can be used to perform the principal components analysis. Using the standard Matlab function one can write

$$
[U, S, V]=S V D(C W T)
$$

where CWT is now the matrix of samples. In particular, the first row of $V$, becomes the discrete approximation to the pseudo power signature of $X$.

It must be noted that this discretization of the CWT does not corresponds with a discrete wavelet transform. For example, the conventional discrete transform created by a multiresolution analysis is only concerned with scales grater than one and tends to ignore the details of a signal. Our discretization enables us to focus on any range of scales, and in fact, for fault detection, the details are often the most useful part of a signal. The most significant issue that arises from using a finite set of scales is that its interpretation as energy distribution must be modified even if the signal can be recovered from the discrete scale transform. To solve this problem, one can propose a discrete scale, discrete time transform.

\subsection{Off-line Flight Fault Detection with SVD Signature: Simulation Result}

For airplane fault detection applications, we assume that normal flight data are available and can be processed to determine a pseudo power signature for normal conditions. During an actual flight we compute the CWT for sensor data and, at any given time, determine the correlation factor with the pseudo power signature. A consistent high correlation will mean that the scale power distribution is similar to that of the pseudo power signature and therefore to a normal flight condition. On the other hand, a small correlation will indicate an abnormal situation.

We report here a typical experiment performed with the B747. The model is trimmed to a given flight condition: straight and level. Out of many input and output variables, we pick deflection of stabilizer as the input, and angle of attack as the output. Then an almost noise free (noise variance: 0.03 ) is given to the input to produce a normal flight condition. In order to create a faulty test flight condition, at some pre-selected time we add a slowly increasing random noise with bigger variance $(0.1)$ to the input. Figure 1 shows the output "angle of attack" for normal flight and test flight conditions. The noise is present from the time step 250, which corresponds to 12.5 seconds. The sampling interval is 0.05 second. Because the noise is slowly increasing, it is hard for a pilot to notice any abnormal change at the moment of onset of fault. Until about the $400^{\text {th }}$ sample point (20 seconds) or later, this change does not appear to be noticeable.

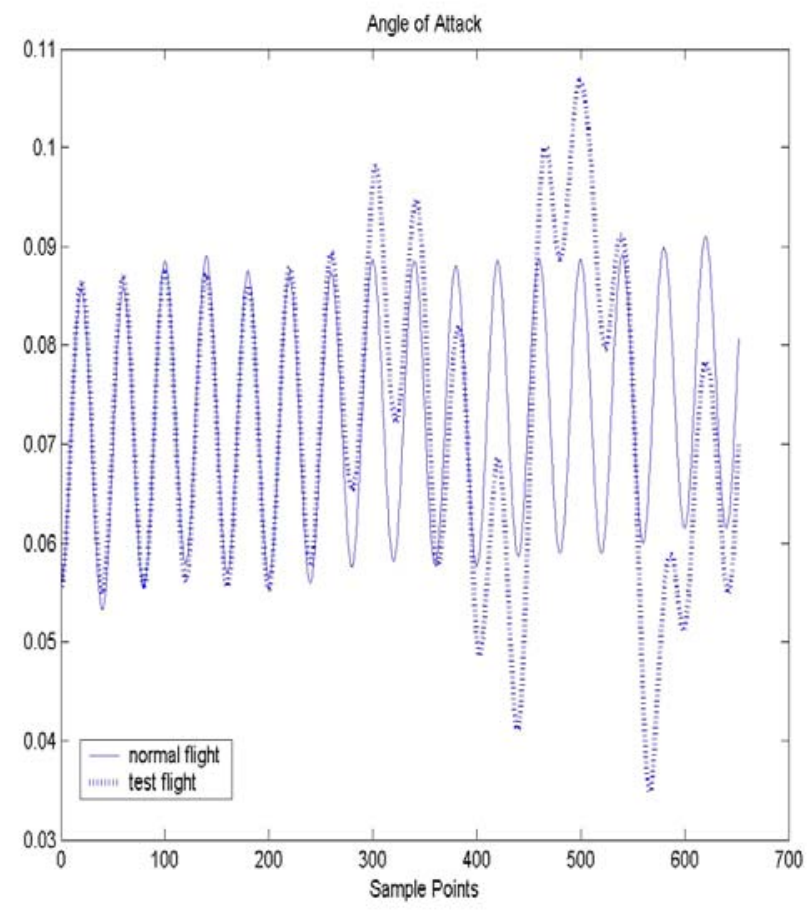

Figure 1. Normal and Test Flight Conditions

Using the matrix SVD technique described earlier, we determine the normal flight signature. We compute the CWT for the test flight and perform the correlation analysis with the normal flight signature. The correlation coefficient is defined and calculated as:

$$
\rho=\frac{\left.<Y \cdot V_{1}\right\rangle}{\|Y\|}
$$


where $Y$ is CWT of the test flight. $V_{1}$ is the first normalized principal vector of the normal flight, i.e. normal flight signature.

Figure 2 illustrates the correlation indicator when the scale range is discretized in 100 values and the wavelet used is Daubechies' Db8. It is apparent that the correlation does not give fast or accurate information about the fault occurrence.

In order to understand the lack of sensitivity of the pseudo power signature it is necessary to examine the distribution of the principal components produced by the SVD. Figure 3 shows all principal singular values for Db8 with 100 scale values. It is obvious that using only the first principal component will produce very poor approximations since there are several singular values that are comparable in value to the first. That result points to the need for approximating the continuous wavelet transform with a sum of separable terms.

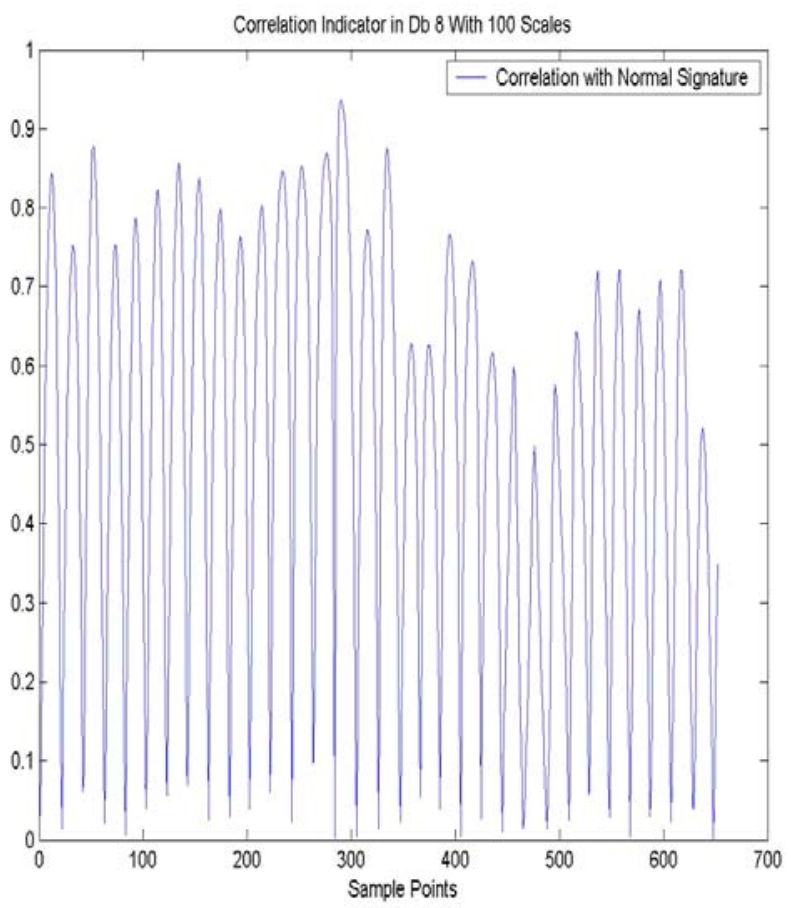

Figure 2. Correlation Indicator in Daubechies 8 with 100 Scale Numbers

\subsection{Refining the SVD Pseudo Signatures: Distance Indicator}

In this section we develop the alternative of improving the pseudo power signatures by considering approximations with more terms. As we shall show, this approach can be considered as defining a "signature subspace." For simplicity we consider only two terms of the approximation in the following, but the extension to more terms is immediately available.

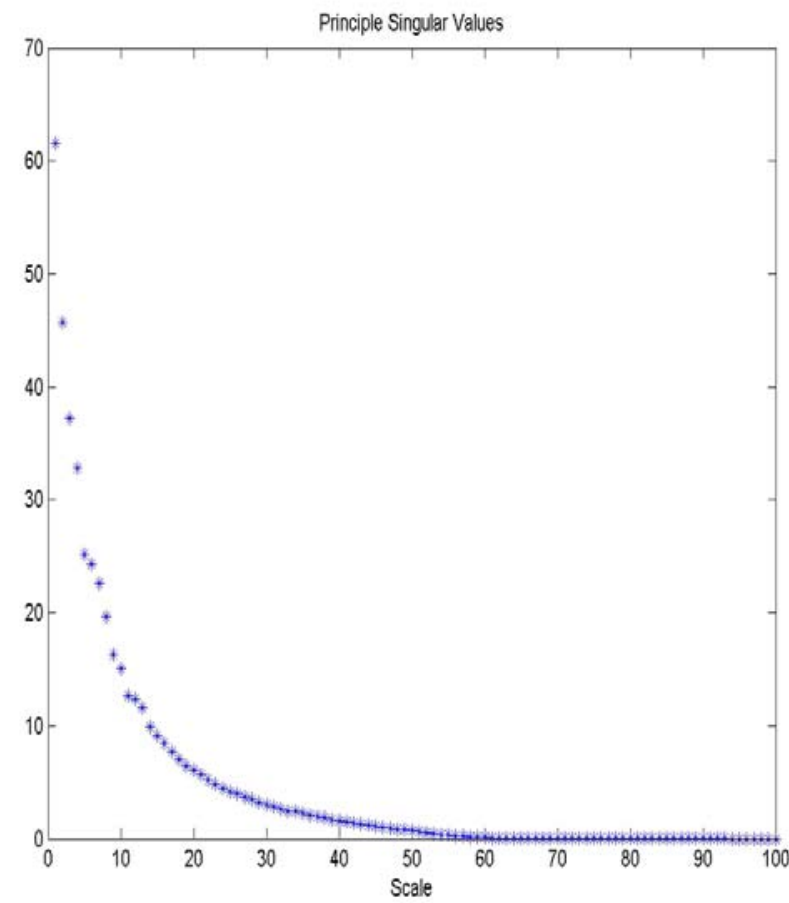

Figure 3. Principal Singular Values of Daubechies 8 with 100 Scale Numbers

Assume then that the CWT can be expressed as

$c_{x}^{\phi}(a, b)=\sigma_{1} s_{1}(a) r_{1}(b)+\sigma_{2} s_{2}(a) r_{2}(b)$

At any time $b_{0}$ one can see that the scale function $c_{x}^{\varphi}\left(., b_{0}\right)$ is a linear combination of the functions $s_{1}(),. s_{2}($.$) . Hence, one can write$

$c_{x}^{\phi}(:, b) \in \operatorname{span}\left\{s_{1}, s_{2}\right\}, \forall b$ 
Moreover, if the vectors have been obtained using singular value decomposition, they are guaranteed to be mutually orthogonal.

Under normal (fault-free) conditions, the CWT should belong to normal signature subspace at all times. Hence the distance to that subspace can be used to measure deviations from normal flight conditions. Eq. (10) (12) illustrate this procedure. Here, $V_{1}$ and $V_{2}$ are the first two normalized principal vectors of the normal flight transform and they form a basis for the subspace of signatures. For any vector, $Y$, the projection of $Y$ on the normal signature plane is of the form $\alpha_{1} V_{1}+\alpha_{2} V_{2}$. The coefficients are determined as projections on the basis vectors can be determined from

$$
\begin{aligned}
& <Y, V_{1}>=\alpha_{1}<V_{1}, V_{1}>+\alpha_{2}<V_{1}, V_{2}> \\
& <Y, V_{2}>=\alpha_{1}<V_{1}, V_{2}>+\alpha_{2}<V_{2}, V_{2}>
\end{aligned}
$$

Since in the SVD the vectors of $V$ are orthogonal to each other, Eq. (10) can be simplified to

$$
\begin{aligned}
& <Y, V_{1}>=\alpha_{1} \\
& <Y, V_{2}>=\alpha_{2}
\end{aligned}
$$

The distance of $Y$ to the normal signature plane is then computed as

$$
d=\sqrt{\|Y\|^{2}-\left(\alpha_{1}^{2}+\alpha_{2}^{2}\right)}
$$

\subsubsection{Simulation Results}

Figure 4 shows the distance indicator using Daubechies' wavelet $\mathrm{Db} 8$ with the scale partitioned in 128 values. It is seen that at the start of fault, the distance from the test flight to the normal signature plane is increasing. This shows that the distance to the subspace indicator provides faster warning of fault than the correlation indicator and raw output data. It is apparent that one can easily increase the dimension of the subspace without a significant increase in the computations. This option could be used to increase the separation between normal and faulty cases and to create distinct subspaces associated to different faults.

In the absence of a model, it is necessary to perform experimental studies to determine the effect of design parameters. Here we have included preliminary experimental data on the effect of the wavelet to be used and the scales to be considered. Different Daubechies' wavelets (from Db4 to Db10) and different scale numbers (from 32 to 128) are experimented for testing sensitiveness of correlation and distance indicators. Figure 5 shows the distance indicator using Daubechies 4 with 100 scale numbers. Distance indicator using Daubechies 8 with 32 scale numbers is shown in Figure 6. And Figure 7 shows the distance indicator using Daubechies 10 with 32 scale numbers. It appears that the type of wavelet to be used is important and we are currently developing criteria for its selection. The scales used are also important and we expect that incorporating minimal information about the system will permit the definition of scale selection criteria. For the cases presented here, one can see that $\mathrm{Db} 8$ with 128 scale numbers is more accurate than the other combinations of Daubechies wavelet length and scale numbers.

A point of concern in Figure 4 is the fact that the distance indicator from test flight to the normal signature plane becomes smaller again after 350 samples points, suggesting that the indicator is more sensitive to the transient created by the onset of the fault than to the faulty situation itself. In order to explore this possibility, we built another faulty test flight condition with fault lasting for a very short time (2 seconds). Figure 8 shows the sensor readings for the faulty test flight and normal flight condition. The test flight shows the faults last in the range of sample point 250 and 350 . Then flight condition is back to normal. Again, we used Daubechies' Db8 with 128 scale numbers to get distance indicator shown in Figure 9. It is easy to see that the distance drop also appears in this case. This result supports the hypothesis that our indicator is detecting the transient. It is therefore necessary to refine the sensitivity of the indicators. For this increase we propose to develop signatures, and subspaces, for faulty conditions and include, in the fault identification, the distance to those various signatures. 


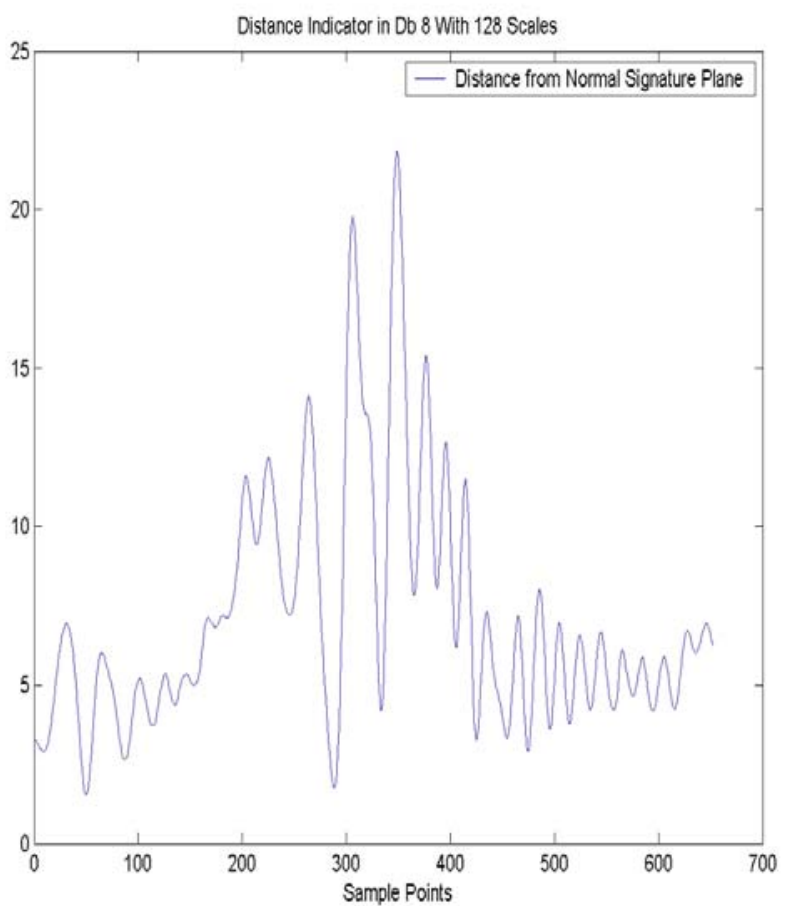

Figure 4. Distance Indicator in Daubechies 8 with 128 Scale Numbers

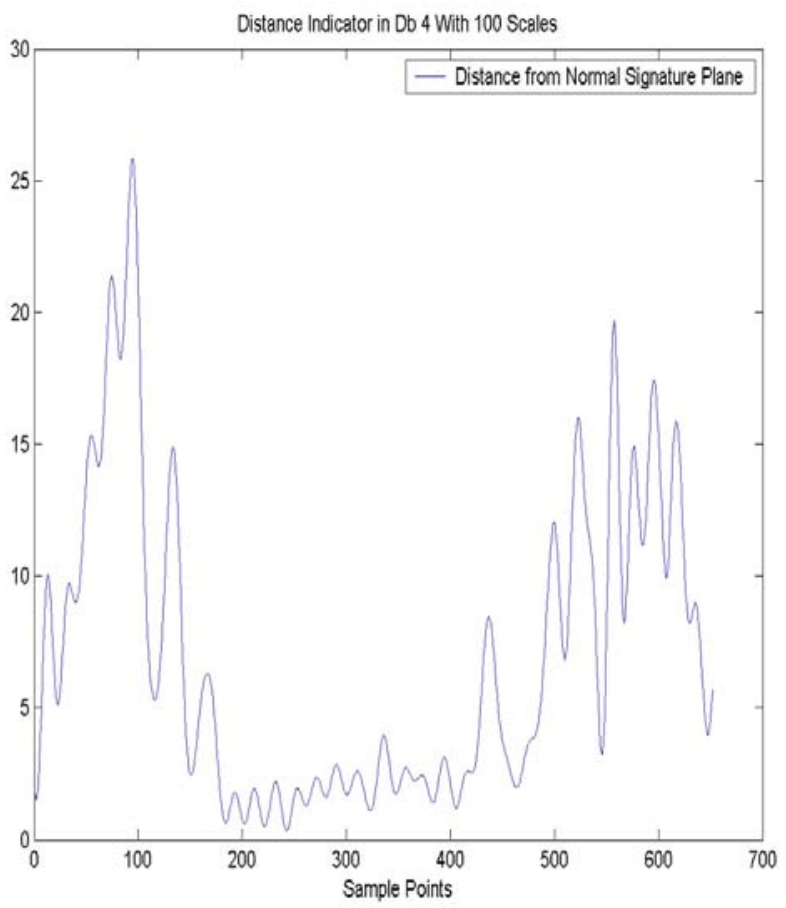

Figure 5. Distance Indicator in Daubechies 4 with 100 Scale Numbers

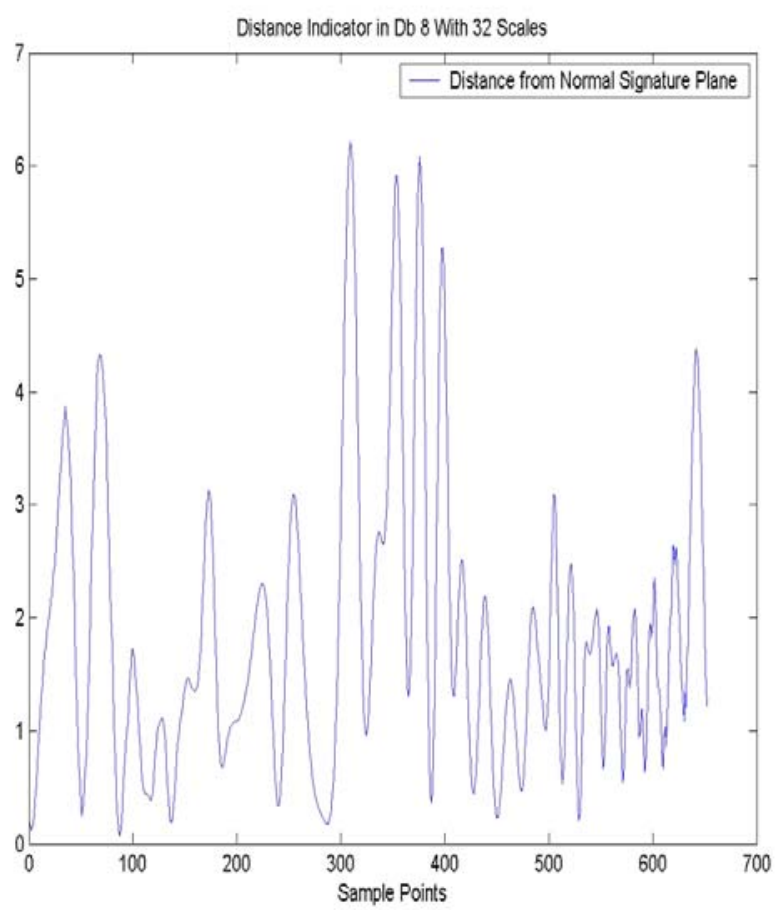

Figure 6. Distance Indicator in Daubechies 8 with 32 Scale Numbers

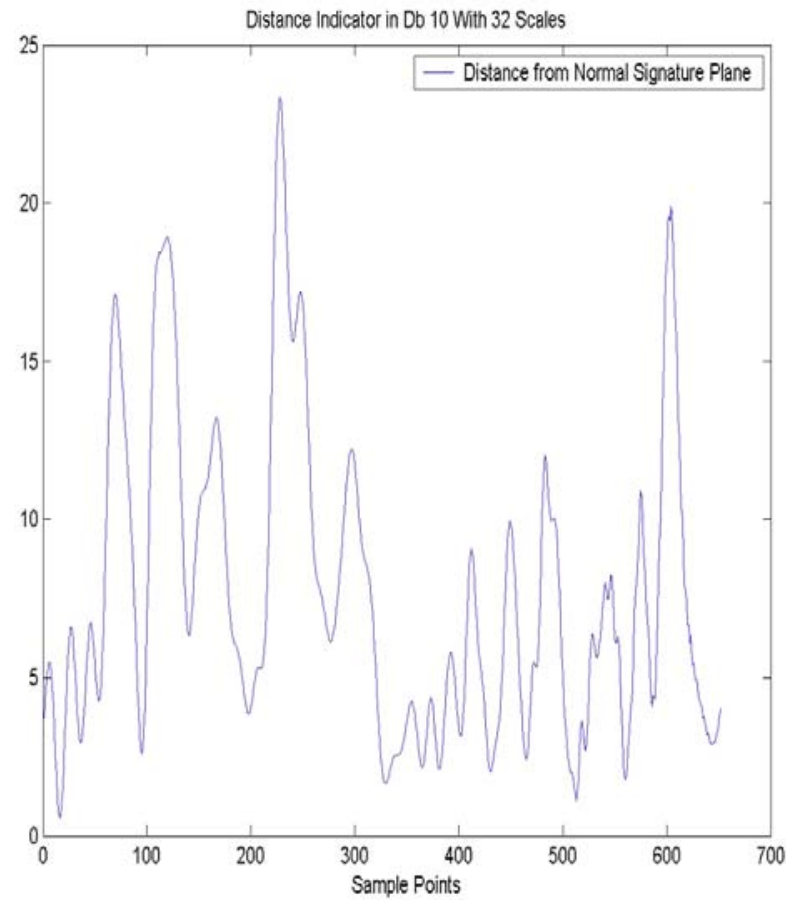

Figure 7. Distance Indicator in Daubechies 10 with 32 Scale Numbers 


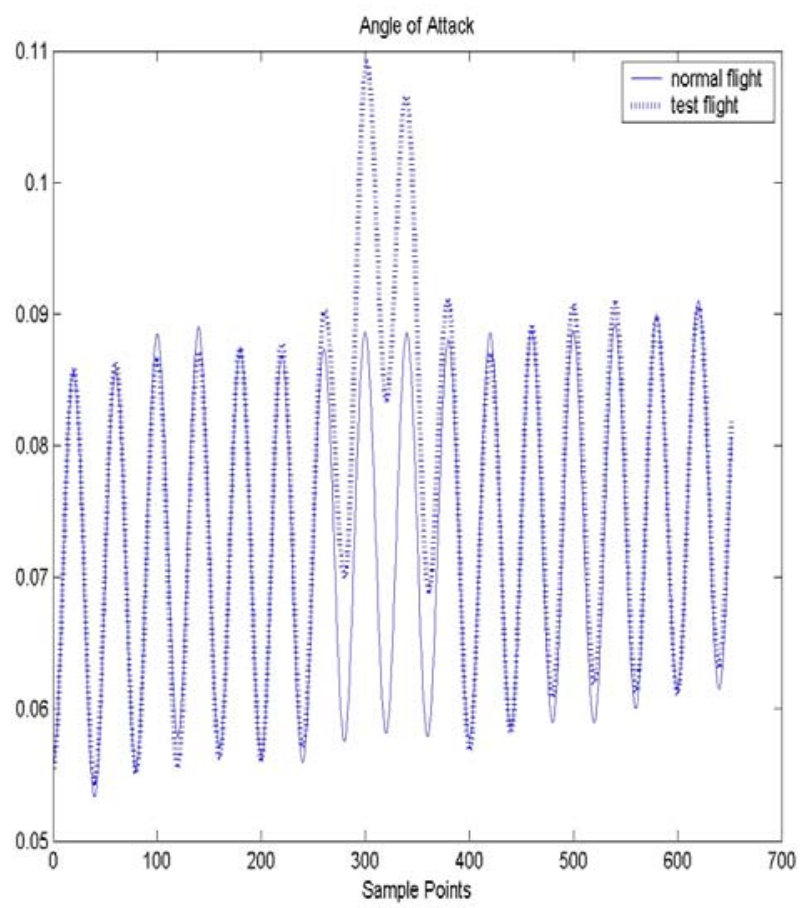

\section{Figure 8. Normal and Test Flight Conditions with Very Short Fault Duration}

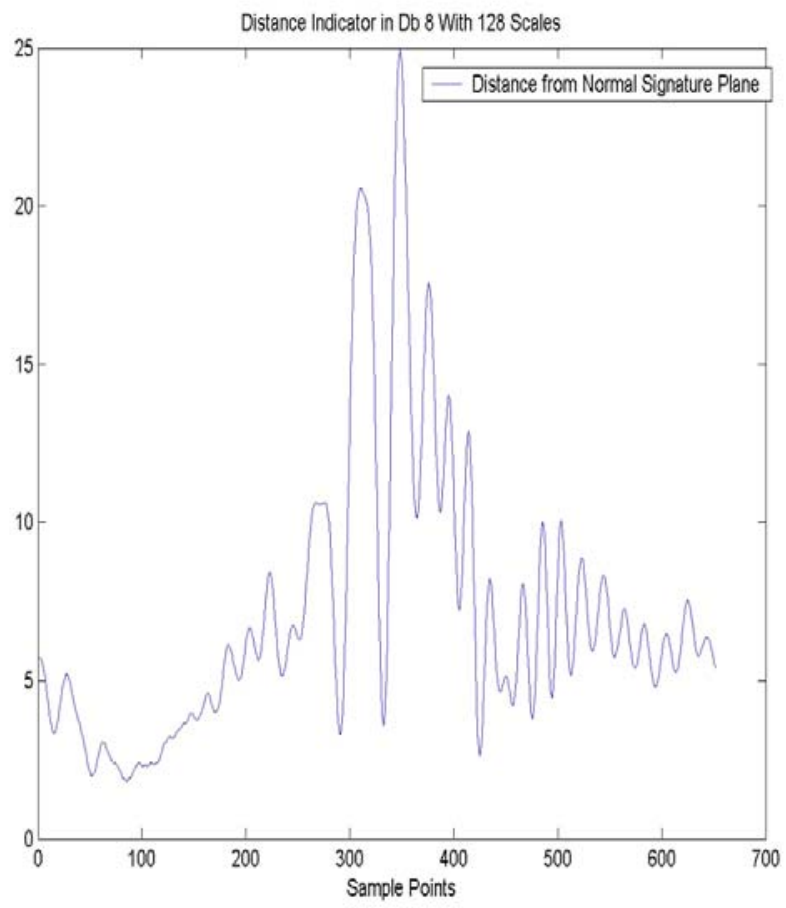

Figure 9. Distance Indicator in Daubechies 8 with 128 Scale Numbers for Very Short Fault Duration

\section{Conclusion and Future Work}

In this paper, we develop further the concept of signatures as tools to detect faulty conditions in a system. We have used the Singular Value Decomposition as the main vehicle to create such signatures. In a very significant manner, we have been able to extend the concept to situations where the principal component is not sensitive enough. We have identified that the situation occurs when the wavelet transform has more than one significant principal value. For those situations we have introduced signature subspaces. In this study we report the use of 2-dimensional signature subspaces, but the principle is easily extended to any number of dimensions, limited only by the number of scales used. Our experimental studies also indicate that the type of wavelet may affect the sensitivity of the indicators. Our future work will continue to explore issues related to the use of signatures for model-free fault detection. In particular: (1) find the reasons for the drop of the distance indicator some time after the onset of fault (see Figure 4). (2) Test different flight conditions with other inputs. (3) Develop criteria for wavelet and scale selection. (4) Develop combined signatures (using singular values, correlation coefficients and distance indicators) for faster and more accurate detection of faults.

Finally, it is essential to point out that the modelfree approach cannot be assumed to be the answer to every situation. Any knowledge of the system under study should be used to produce better fault indicators. In this sense, the use of DSP techniques reduces the amount of detailed information that is necessary in residue-based approaches.

\section{Reference}

[1] Wald, A. 1947, Sequential Analysis, New York: Wiley

[2] Basseville Michèle and Igor V. Nikiforov, 1993, Detection of Abrupt Changes - Theory and Application, Prentice-Hall, Inc.

[3] Pei X and F.N. Chowdhury, Aug 1999, Unsupervised Neural Networks for Fault Detection and Classification in Dynamic 
Systems, Proc. IEEE International Conference on Control Applications (CCA99), pp. 640 645

[4] Montgomery R. C. and D. B. Price, Feb 1976, Failure Accommodation in Digital Flight Control Systems Accounting for Nonlinear Aircraft Dynamics, AIAA Journal of Aircraft, Vol.13, No.2

[5] Montgomery R .C. and A.K. Caglayan, Feb 1976, Failure Accommodation in Digital Flight Control Systems by Bayesian Decision Theory, AIAA Journal of Aircraft, Vol.13, No.2

[6] Chowdhury F and J.L. Aravena, Sep 1998, A Modular Methodology for Fast Fault Detection and Classification in Power Systems, IEEE Trans. Control Sys. Tech., Vol. 6, No. 5, pp. 623 - 634

[7] Aravena Jorge L, and F. Chowdhury, Oct 2001, Fault Detection of Flight Critical Systems, Proc. $20^{\text {th }}$ Digital Avionic Systems, Daytona Beach

[8] Van Der Linden, C.A.A.M., 1996, DASMAT

- Delft University Aircraft Simulation Model and Analysis Tool. A Matlab/Simulink Environment for Flight Dynamics and Control analysis, Report LR-781, Delft University of Technology, Delft

[9] Venkatachalam Vidya, 1998, Pseudo Power Signatures for Nonstationary Signal Analysis and Classification, $\mathrm{PhD}$ Dissertation, Louisiana State University 